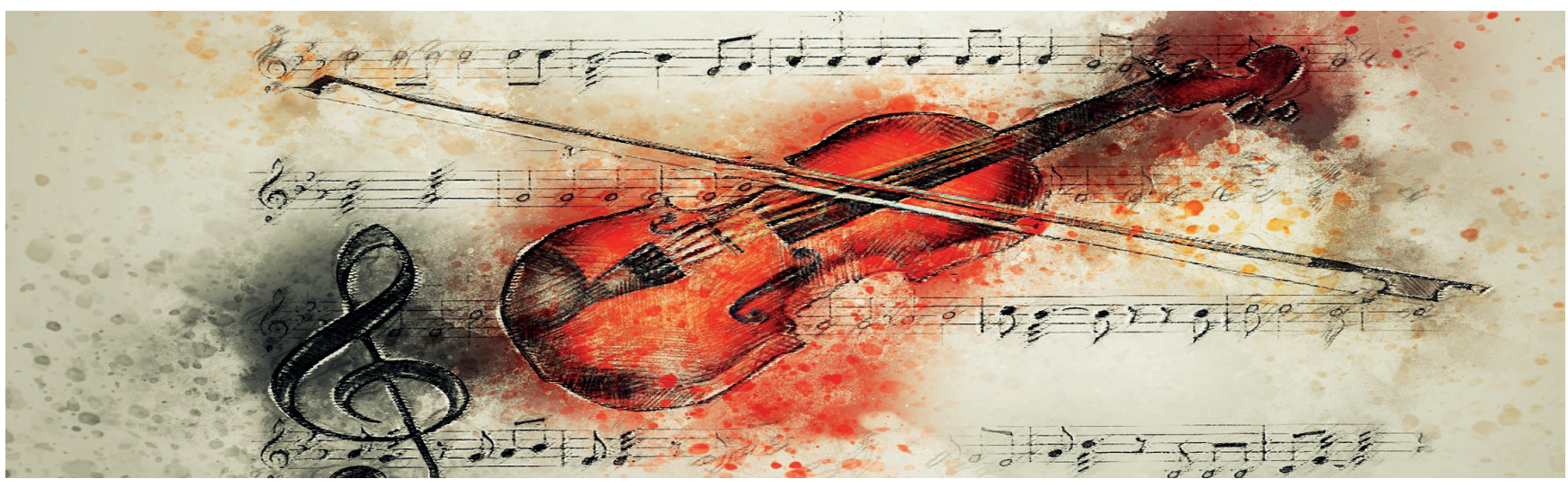

Tomado dehttps://pixabay.com/es/illustrations/violín-música-notas-arte-resumen-2412357/

\title{
Formación artística y cultural en la educación secundaria obligatoria: los casos de Francia y México
}

\section{Artistic and cultural training in compulsory secondary education: the cases of France and Mexico \\ Ileana Rojas-Moreno*, Patricia Ducoing-Watty}

\section{RESUMEN}

Este artículo se basa en los resultados del proyecto de investigación institucional PAPIITUNAM IN401218 sobre la educación secundaria obligatoria en trece países (9 latinoamericanos, 4 europeos). Retomamos aquí uno de sus objetivos: destacar las semejanzas y diferencias en la Educación Artística en el currículum prescrito en dos de los países de la muestra (Francia y México). Para este efecto empleamos una estrategia metodológica de corte comparativo, a fin de argumentar la vinculación entre políticas educativas internacionales y currículos nacionales, en un contexto caracterizado por tendencias mundiales de internacionalización, obligatoriedad y estandarización de la oferta educativa. Respecto de la Educación Artística y su papel en el logro de una mejor calidad de la educación, ubicamos su contribución formativa para atender las necesidades de creatividad y sensibilización cultural en el siglo XXI.

Palabras clave: educación secundaria obligatoria, educación artística, currículum prescrito, educación comparada.
ABSTRACT

This article is based on the results of the institutional research project PAPIIT-UNAM IN 401218 on compulsory secondary education in nine countries in Latin America and four in Europe. Here we return to one of its objectives, aimed toward highlighting the similarities and differences in Artistic Education in the curriculum prescribed in two of the countries in the sample (France and Mexico). For this purpose we use a comparative methodological strategy, with the intention of arguing the link between international educational policies and national curricula, in a context characterized by global trends in internationalization, mandatory and standardization of the educational offer. Regarding Artistic Education and its role in achieving a better quality of education, we place its formative contribution in meeting the need of both creativity and cultural awareness in the 21st century.

Keywords: Compulsory secondary education, arts education, prescribed curriculum, comparative education. 
Presentación y encuadre teórico-metodológico Históricamente, la incorporación de la educación artística en el ámbito escolar se ha caracterizado por tendencias curriculares de corte academicista cuyos rasgos más notables han delimitado un espectro de enseñanzas y aprendizajes enfocado en la adquisición de destrezas para el dibujo, la danza, el canto, el solfeo y el uso de un instrumento musical específico. A lo largo de poco más de dos siglos, la participación de educadores y artistas profesionales en la definición de un área específica en el marco de planes y programas de estudios de la educación obligatoria ha dado lugar a la conformación de un saber acumulado en el que confluyen, por una parte, visiones disciplinarias sobre el arte en sus diferentes manifestaciones de creatividad y, por otra, modelos pedagógicos centrados en la figura del docente mediante los cuales se ha buscado fundamentar una normatividad para la enseñanza de conocimientos de esta naturaleza (Aguirre, 2008; Jiménez, Aguirre y Pimentel, 2009).

Así, en la planeación de los currículos oficiales de la educación primaria de diferentes países del orbe se ha optado por atender la formación artística mediante la delimitación de una única área de conocimiento, si bien en el caso de la educación secundaria obligatoria se convirtió en un modelo más de corte disciplinar; el énfasis por utilizar abordajes globales, no obstante la especificidad en el estudio de los diferentes ámbitos artísticos, se convirtió en una estrategia académico-administrativa usual para la incorporación e integración de contenidos. Actualmente, la experiencia y el avance del conocimiento han puesto en cuestión la eficacia de estas decisiones al destacar condiciones a resolver como la limitada relevancia concedida a la formación artística, en contraste con áreas académicas como las de ciencias, lenguaje y tecnologías, o bien, la escasa atención a la formación de profesores especializados en las ramas comprendidas en el estudio del arte y la cultura.

Por ejemplo, en las agendas de política educativa internacional -específicamente de las dos últi- mas décadas- se ha concedido una atención especial al aprendizaje del arte y de la cultura en las instituciones de educación obligatoria, al considerar que la formación artística y cultural constituye una de las estrategias de mayor influencia para la construcción de una ciudadanía intercultural. En opinión de expertos (Ruiz, y Santos, 2009) y de acuerdo con la panorámica educativa planteada por diversos organismos internacionales (UNESCO, 2006; EACEA P9/ Eurydice, 2009; Ferro, Wagner, Veloso, IJdens, y Teixeira, 2019), la inclusión del arte en la educación, a través de un área curricular destinada a la educación artística y mediante la educación por el arte, coadyuva en el desarrollo integral de niños y jóvenes. Asimismo, las acciones emprendidas por organismos y/o entidades internacionales (UNESCO, Organización de Estados Iberoamericanos para la Educación, la Ciencias y la Cultura, OEI, Red Eurydice) a través de reuniones cumbre, congresos, convenciones y encuentros, representan un movimientos de avance mundial en favor de la construcción de un marco normativo integral tendente a producir diversos recursos de soporte (diagnósticos, estadísticas, mapas e inventarios nacionales y regionales) sobre acervos y experiencias artístico-culturales. Este conjunto de dinámicas ha tenido como propósito central el de influir en la toma de decisiones políticas de ministerios y planificadores de los sistemas educativos nacionales, a fin de integrar la educación artística en el conjunto de agendas de políticas públicas nacionales.

A partir de este encuadre y para efectos del presente artículo, recuperamos los resultados de base documental, de una investigación institucional de corte comparativo sobre la educación secundaria obligatoria en países latinoamericanos y europeos (PAPIIT IN-401218), a fin de enfocarnos básicamente en destacar algunos rasgos de contraste de esta área formativa en los currículos oficiales de dos de los países de la muestra (casos Francia y México), seleccionados por dos motivos. En primer lugar, por el interés de apoyarnos en una mirada internacional de encuadres regionales contrastantes como 
son la Unión Europea y la región latinoamericana; en segundo lugar, por la divergencia entre ambos países en cuanto a los contextos socioeconómicos, las políticas públicas y su vinculación con los modelos académicos implantados. Para tal propósito y a partir de una contextualización sociodemográfica inicial hemos incluido los rubros de estructura y organización de sistemas educativos con especial interés en el nivel de la educación secundaria obligatoria, para llegar a la caracterización de la formación artística y cultural en dicho nivel educativo, considerando una puntualización abreviada de referentes como políticas educativas, contenidos curriculares y formación de docentes. Nuestras preguntas de investigación fueron las siguientes: a) ¿cuáles son las tendencias internacionales a propósito de la formación artística y cultural en la educación secundaria obligatoria?; y, b) de acuerdo con el contexto sociohistórico y económico de cada país, ¿cuáles son los elementos comunes y las diferencias específicas en la formación académica de referencia en este nivel educativo?

Para el desarrollo aquí propuesto elegimos como categoría central de análisis la de "formación artística y cultural en la educación secundaria obligatoria”, para nombrar la formación básica sobre las artes y la herencia cultural que se propicia en entornos escolares de este nivel educativo (Aguirre y Giráldez, 2009, p.75), misma que utilizamos como el eje articulante entre la revisión de los casos nacionales y su vinculación con el panorama internacional. En cuanto a los planteamientos de soporte para dicha categoría y el entretejido con la aproximación comparativa a nuestro objeto de estudio, destacamos aquí algunos referentes. El primero de ellos se deriva de los planteamientos de Marsch y Willis (citados por Adamson, y Morris, 2010, p.322), para situar cinco vertientes del currículum: a) herencia clásica organizada en asignaturas tradicionales; b) avance del conocimiento agrupado en ramas, ciencias o disciplinas que posibilitan la vida institucional; c) utilidad social y económica de conocimientos y habilidades adquiridos; d) planeación de pro- ductos de aprendizaje; y, e) logros individuales de docentes y alumnos. Como segundo referente retomamos los aportes de Casimiro (2015, p.50) sobre el vínculo entre política y currículum y su aplicación en la implantación nacional y contextualizada de un modelo educativo específico, pero vinculado con lineamientos universalizantes. El tercero y último referente atiende la estrategia metodológica comparativa, basada en los planteamientos de Schriewer (1993; 2011) sobre el entramado de los casos nacionales y el contexto de los procesos de internacionalización de sistemas, niveles y modelos educativos.

Con base en este acercamiento nos interesa mostrar que, no obstante los avances tanto en el conocimiento sobre el papel primordial de la educación artística en la preparación integral de niños y jóvenes, como en los esfuerzos de las comunidades internacionales por incidir en las agendas de políticas nacionales en favor de una educación de calidad y para la ciudadanía en el nuevo siglo, las diferencias entre los casos analizados dan cuenta no solo del peso definitorio que tienen los contextos sociodemográficos nacionales, sino además de las estrategias de intervención estatal a través de la participación de tomadores de decisiones y/o planificadores responsables de la puesta en marcha de políticas públicas, ya sea para impulsar una formación artística de calidad, o bien, para incluirla como una asignatura más del currículo oficial del nivel en cuestión.

1. Contextualización sociodemográfica abreviada de los casos nacionales

Para situar el ejercicio de contraste de la educación artística en el nivel de educación secundaria obligatoria en los casos de Francia y México, tomamos como punto de partida un panorama sociodemográfico abreviado que -a manera de esbozo- incluye algunos datos estadísticos de soporte que evidencian las particularidades nacionales en cuanto a condiciones socioeconómicas y educativas. El primer conjunto de datos nos muestra de entrada dos diferencias importantes a destacar: la primera 
tiene que ver con el rubro del Producto Interno Bruto (PIB) según el cual México se encuentra muy por debajo del promedio de 25,908 dólares establecido por la Organización para la Cooperación y el Desarrollo Económico (OECD, por sus siglas en inglés), quedando así en el lugar 90 del ranking mundial mientras que Francia, con una diferencia de cincuenta puntos, está situada en el lugar 40. La segunda diferencia se refiere al porcentaje de población ubicada por debajo del umbral de pobreza que, nuevamente en el caso mexicano, un poco menos de la mitad de la población total se encuentra en una situación económica desventajosa (Tabla 1). En el segundo conjunto de datos ubicamos la relación entre el número total de población, la población comprendida en el rango de edad de 0 a 14 años y de ese mismo rango la población escolarizada. Aquí destacamos entre ambos países las diferencias entre la proporción de este grupo de edad de la población, con un $18.48 \%$ en Francia frente a un $26.61 \%$ en México. En cuanto a la población escolarizada para ese mismo rango de edad, el contraste observado nos muestra que en Francia casi el $100 \%$ de ese grupo poblacional forma parte del sistema educativo, mientras que en México la población escolarizada en ese mismo rango ocupa las cuatro quintas partes de la población total. De este mismo grupo, otros datos importantes de contraste son los concernientes al gasto educativo, la ex-

\begin{tabular}{|c|c|c|}
\hline Rubro/País & Francia & México \\
\hline Localización & $\begin{array}{c}\text { Territorio metropolitano en Europa } \\
\text { Occidental y territorio de ultramar } \\
\text { (América, Océanos Atlántico, Pacífico e } \\
\text { Índico, Antártida) }\end{array}$ & $\begin{array}{l}\text { Parte meridional de América del } \\
\text { Norte }\end{array}$ \\
\hline $\begin{array}{c}\text { Superficie territorial } \\
\text { total }\end{array}$ & $675,417 \mathrm{~km}^{2}$ & I,964,375 km² \\
\hline $\begin{array}{c}\text { Sistema político } \\
\text { PIB nominal Per } \\
\text { cápita (2018) (dólares } \\
\text { corrientes) }\end{array}$ & República semipresidencialista & República federal presidencial \\
\hline $\begin{array}{c}\text { Lugar en el mundo } \\
\text { IDH (2018) }\end{array}$ & 45,025 & 10,283 \\
\hline $\begin{array}{c}\text { Población con } \\
\text { ingresos por debajo } \\
\text { del umbral de pobreza } \\
\text { (2018) }\end{array}$ & 40 & 90.774 \\
\hline $\begin{array}{c}\text { Idioma oficial } \\
\text { Religiones }\end{array}$ & Francés/Lenguas de Francia & Español/67 lenguas indígenas \\
\hline No hay una religión oficial & No hay una religión oficial \\
\hline
\end{tabular}

Elaborado por las autoras con datos de Central Intelligence Agency (2019) The world factbook. Francia y México (https://www.cia.gov/library/publications/the-world-factbook/).

Tabla 1. Comparativo de datos demográficos, políticos y socioeconómicos (2018).

Table 1. Comparison of demographic, political and socioeconomic data (2018). 
pectativa de vida escolar y el lugar que ocupa cada país según los resultados del Programa de Evaluación Internacional de Estudiantes (PISA, por sus siglas en inglés) de la OECD, PISA (2018). Desde esta perspectiva observamos una condición de desarrollo educativo más avanzada en Francia, que contrasta con las evidentes desventajas que prevalecen en el caso mexicano (Tabla 2).

\section{Sistemas educativos y delimitación etaria de la educación secundaria obligatoria}

A modo de caracterizar los rasgos básicos de la educación secundaria obligatoria en Francia y
México, iniciaremos con la estructura y la organización actuales de los sistemas educativos nacionales de referencia, tomando como base la Clasificación Internacional Normalizada de la Educación (UNESCO/Cine, 2011) sobre la distribución de ciclos, niveles, grados y grupos de edad, y sin perder de vista que en cada caso la conformación del propio sistema educativo reúne sus particularidades históricas, político-administrativas e institucionales. No obstante estas diferencias, es evidente que ambos países comparten elementos comunes a propósito de los agrupamientos para los niveles 0 de Educación de la primera infancia y 1 de Primaria,

\begin{tabular}{|c|c|c|c|}
\hline \multicolumn{2}{|c|}{ Rubro/País } & Francia & México \\
\hline \multicolumn{2}{|c|}{ Población total (2018) } & $67,364,357$ & $125,959,205$ \\
\hline \multicolumn{2}{|c|}{ Población de 0 a 14 años (total y porcentaje) (2018) } & $12,449,518(18.48 \%)$ & $33,521,433(26.61 \%)$ \\
\hline \multicolumn{2}{|c|}{ Población de 0 a 14 años escolarizada (2018) } & $12,345,313(99.16 \%)$ & $27,675,167$ (82.56\%) \\
\hline \multicolumn{2}{|c|}{ Gasto en educación } & $5.5 \%$ del $\mathrm{PPB}$ & $5.2 \%$ del PPB \\
\hline \multicolumn{2}{|c|}{$\begin{array}{c}\text { Expectativa de vida escolar incluyendo preescolar, } \\
\text { primaria y secundaria baja (años) (2018) }\end{array}$} & 12.4 & 9.9 \\
\hline \multirow{4}{*}{$\begin{array}{c}\text { Resultados de } \\
\text { evaluación PISA } \\
\text { (2018) }\end{array}$} & Lectura & 493 & 420 \\
\hline & Matemáticas & 495 & 409 \\
\hline & Ciencias & 493 & 419 \\
\hline & Ranking & $23^{\circ}$ & $53^{\circ}$ \\
\hline
\end{tabular}

Tabla elaborada por las autoras con datos de:

1) Central Intelligence Agency (2019) The world factbook. Francia y México. (https://www.cia.gov/ library/publications/the-world-factbook/).

2) Banco Mundial (2019)

(https://databank.worldbank.org/indicator/UIS.SLE.0?id=c755d342\&report_name=EdStats_Indicators_Report \&populartype=series).

3) Organización para la Cooperación y el Desarrollo Económicos (OECD), PISA, 2018 Database http://www.oecd.org/pisa/data/2018database/.

Tabla 2. Comparativo de población total, población de 0 a 14 años, parte proporcional de población de 0 a 14 años escolarizada (2018 y resultados de evaluación de pisa (2018).

Table 2. Comparison of total population, population aged 0 to 14 years, proportional share of population aged 0 to 14 years enrolled in school (2018) and pisa evaluation results (2018). 
en lo concerniente a grupos etarios y obligatoriedad. Las variaciones se observan en cuanto a la duración y las denominaciones oficiales del citado nivel (Tabla 3). Respecto de la educación secundaria obligatoria y teniendo como criterio la demarcación etaria de la edad ideal (11 - 14 años), observamos una denominación diferenciada que remarca, en ambos casos, el avance respecto de un nivel elemental (Francia: "Segundo nivel Collège-Secundaria y Liceo", México: “Educación Secundaria”). Entre ambos países contrasta también una duración diferenciada (Francia: 4 años; México: 3 años) a la vez que se comparte el hecho de que este nivel educativo tenga un carácter vestibular dado que represente el enlace con los ciclos subsiguientes. Una vez perfilado el encuadre anterior, veamos más puntualmente y a modo de contraste las características más sobresalientes del área que nos ocupa.

\section{Aproximación comparativa de la forma- ción artística y cultural en educación secun- daria obligatoria}

En términos de referirnos a la formación académica sobre las artes y la cultura que se propicia en las instituciones educativas del nivel secundario, las distinciones entre los países seleccionados aluden necesariamente al papel del Estado como responsable directo de la educación oficial. Por ejemplo, en Francia la educación artística está ligada a la educación cultural, y al referirse a la categorización de "artística" siempre se le acompaña de "y cultural". Cabe señalar que no se trata de una denominación novedosa, dado que la atención estatal de la educación artística y cultural es un referente de larga data ubicado en la intervención del Estado a través de los ministerios encargados de la Cultura y de la Educación Nacional, así como por los organismos de los diferentes servicios desconcentrados: las Direcciones Regionales de Asuntos Culturales (DRAC), entidades establecidas en los diferentes departamentos, además de los rectorados de las diversas academias en todo el territorio francés.

En contraste, en México la Secretaría de Cultura (anteriormente denominado Consejo Nacional para la Cultura y las Artes/CONACULTA) y la Secretaría de Educación Pública (SEP) funcionan como entidades gubernamentales independientes, pues de hecho hasta 2015 la SEP se vinculaba con el citado CONACULTA como un órgano desconcentrado a cargo de la educación y la promoción de la cultura y las artes. Con la reciente creación de la referida Secretaría de Cultura, esta entidad gubernamental quedó a cargo de la implantación de políticas, programas y proyectos de expresión y difusión de la cultura y las artes, sin haberse definido su relación con el ramo educativo. En la actualidad, mientras la Secretaría de Cultura se destina a promover y difundir las expresiones culturales y artísticas, y la presencia del país en el extranjero, la SEP es la entidad oficialmente responsable de la educación artística en el marco del sistema educativo, en particular en el ámbito de la educación obligatoria. Veamos a continuación algunos rasgos definitorios de la formación académica en educación artística, concretamente en los rubros de políticas educativas, contenidos curriculares y formación de docentes, en los casos estudiados.

\subsection{Políticas de educación artística y cultural en Francia}

Desde la perspectiva de las políticas educativas, para el Estado francés la educación artística y cultural es un asunto prioritario que viene consolidándose en el marco de la democratización y con base en principios de corte filosófico-político tales como igualdad de acceso a las prácticas artísticas y culturales para todos en la escuela y fuera de ella (en la familia, en la comunidad, en la sociedad en general), a través de los establecimientos escolares y de las estructuras y equipamientos culturales de los diversos territorios. De acuerdo con En el (2011), la consolidación de la educación artística y cultural en Francia ha sido posible gracias a tres movimientos desarrollados en los últimos tiempos: 1) el crecimiento y la diversificación de proyectos apoyados en el incremento del financiamiento público; 2) la mejora de la oferta a través de múltiples vías de toda la cadena de propuestas de 


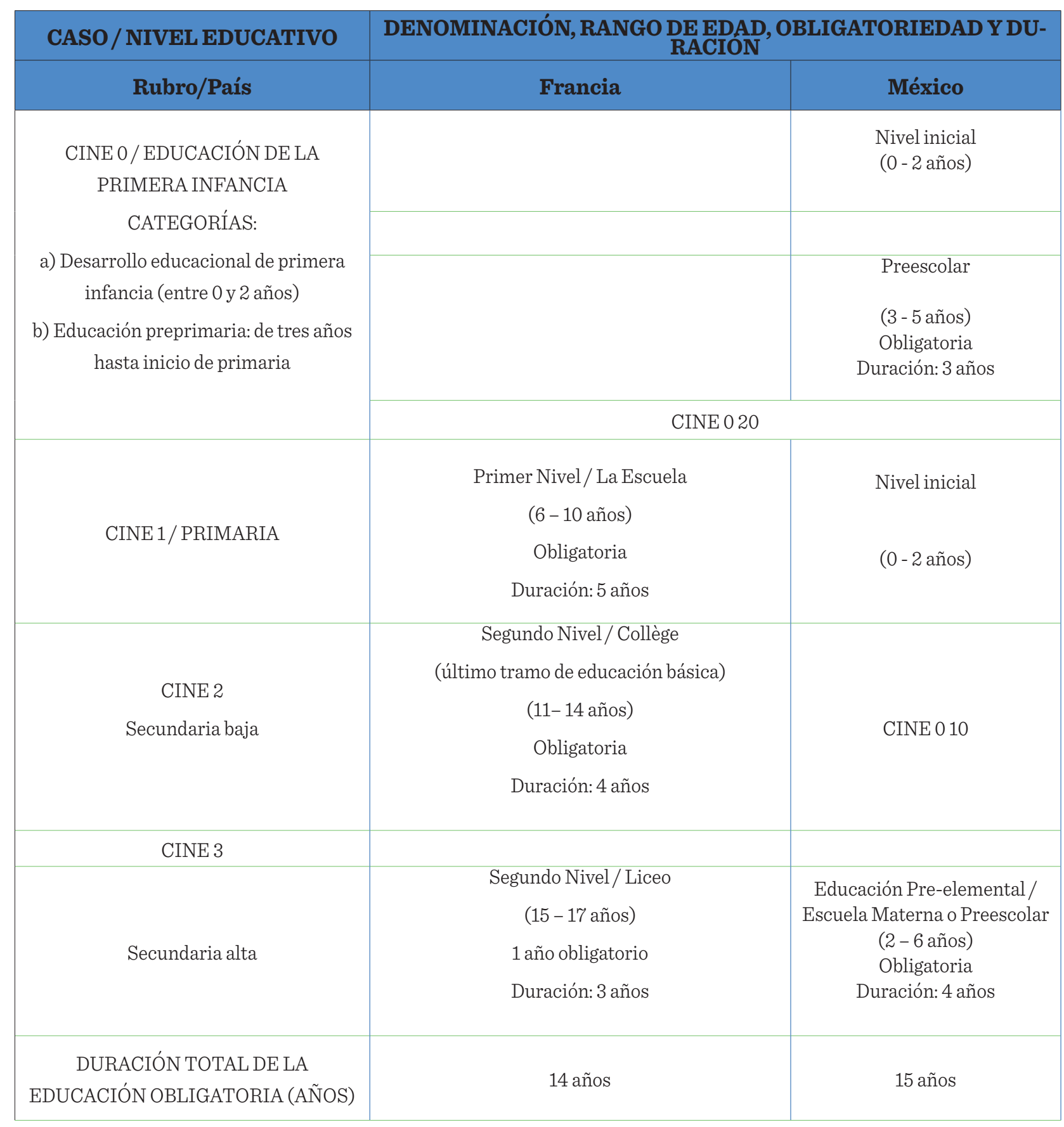

Tabla elaborada por las autoras con datos de:

1) Unesco/Cine (2011) (https://unesdoc.unesco.org/ark:/48223/pf0000220782).

2) Ministère de l’Éducation Nationale et de la Jeunesse (2019) (https://eduscol.education.fr/ cid86943/le-socle-commun.html).

3) Sistema de Información de Tendencias Educativas en América Latina (Siteal (2019) (http://www. siteal.iipe.unesco.org/sites/default/assets/pdf/educacion/siteal_ed_mexico_20180802.pdf).

Tabla 3. Estructura y organización de la educación obligatoria en los sistemas educativos de Francia y México.

Table 3. Structure and organization of compulsory education in the educational systems of France and Mexico. 
educación artística y cultural, que van desde el trabajo en las aulas por medio de proyectos y en su vinculación con otros aprendizajes escolares, así como la mejora de los espacios destinados a la difusión y la práctica de diversas actividades; y, 3) la ampliación y diversificación de los actores involucrados y el establecimiento de estrategias de colaboración entre artistas, estructuras culturales, establecimientos escolares, instituciones. Además, hay que considerar la oferta de programas para acceder a la formación en diversos ámbitos de la educación artística y cultural (République Française. Ministère de la Culture, s/f).

En relación con este señalamiento, en la hoja de ruta interministerial definida en 2015 por los Ministerios de la Educación Nacional, de Enseñanza Superior e Investigación, y el de Cultura y Comunicación, se formularon los objetivos comunes relativos a la formación artística y cultural, documento al que se añadió en 2016 la "Carta para la educación artística y cultural", estableciéndose entre otros, los siguientes principios clave: la Educación Artística y Cultural (EAC) debe ser accesible a todos, particularmente a los jóvenes; la EAC promueve el encuentro con artistas y la frecuentación de las obras y lugares culturales; la EAC pretende la adquisición de una cultura compartida diversificada; la EAC contribuye a la formación y emancipación de las personas y a la formación ciudadana; la EAC permite a los jóvenes dar sentido a sus experiencias (République Française, Ministère de l'Éducation Nationale, Ministère de la Culture et de la Communication et autres, 2016).

\subsection{Políticas de educación artística y cultural en México}

En la actual administración no se han definido aún las políticas artísticas y culturales, como tampoco se cuenta con un Programa Nacional de Cultura a cargo de la Secretaría de Cultura. Hasta el momento presente el único planteamiento en este sentido ha quedado indicado en el Plan Nacional de Desarrollo 2019-2024 en los siguientes términos: “... nadie debe ser excluido de las actividades y los circuitos de la cultura, los cuales representan, en la actual circunstancia, factores de paz, cohesión social, convivencia y espiritualidad. [...] el gobierno federal priorizará en este [rubro] las necesidades de los sectores más marginados, indefensos y depauperados, e impulsará una vigorosa acción cultural en las zonas más pobres del país (Presidencia de la República, 2019, p.46). Otro señalamiento importante en este sentido puntualiza que no se descuidarán las funciones que tradicionalmente ha tenido bajo su responsabilidad la Secretaría de Cultura, tales como la difusión, el enriquecimiento y la consolidación de la diversidad cultural. No obstante, a partir de 2018 el gobierno federal ha emprendido una serie de recortes presupuestales siendo el caso del sector cultural como uno de los más afectados con un recorte de alrededor de mil millones de pesos, contando únicamente con un presupuesto que representa el porcentaje más bajo de las tres últimas administraciones.

Cabe señalar también que durante la administración antepasada (2007-2012) se contaba con un Programa Nacional de Cultura y varios ejes de política cultural, entre los que destacaban los de patrimonio y diversidad cultural, infraestructura cultural, promoción cultural nacional e internacional, estímulos públicos a la creación y mecenazgo, formación e investigación antropológica, histórica, cultural y artística, esparcimiento cultural y lectura (Secretaría de Cultura, 2015, s/p). Esto último al buscar un fortalecimiento de las relaciones entre la SEP y la de Secretaría de Cultura a través de políticas y programas tendentes a “...fomentar en los estudiantes el reconocimiento, el aprecio y la experiencia formativa de las diversas manifestaciones culturales y del arte" (Gobierno de la República, Secretaría de Educación Pública, Secretaría de Cultura, 2017, p.8).

Este esfuerzo conjunto se concretó en una convocatoria dirigida a las instituciones educativas, a los docentes y a la sociedad en general a valorar la educación artística y cultural tanto en los espacios escolares, como fuera de los mismos (Gobierno de la República, Secretaría de Educación Pública, Secretaría de Cultura, 2017, p.8). 


\subsection{Contenidos curriculares para la formación artística y cultural en Francia}

El currículum francés de la educación obligatoria está organizado con base en un zoclo común de conocimientos, de competencias y de cultura, en el que se puntualizan las adquisiciones básicas que todos los alumnos deben lograr al finalizar cada ciclo escolar. Por otra parte, también se ha estructurado en cinco ámbitos: 1) los lenguajes para pensar y comunicar; 2) los métodos y las herramientas para aprender; 3) la formación de la persona y del ciudadano; 4) los sistemas naturales y los sistemas técnicos; y, 5) las representaciones del mundo y de la actividad humana (Ministère de l'Education Nationale et de la Jeunesse, 2019, s/p). Respecto de la educación artística, esta se encuentra ubicada en tres de los ámbitos del zoclo común: en el de los lenguajes, en el relativo a los sistemas naturales y técnicos, y en el correspondiente a las representaciones del mundo y de la actividad humana, lo que denota que esta formación es abordada desde una perspectiva interdisciplinar.

En el caso del nivel de educación secundaria obligatoria también llamada Collège (Colegio), el currículum abarca cuatro años distribuidos en ciclos: el sexto grado, equivalente al primero del Collège corresponde al último año del ciclo 3 o de consolidación; el quinto, el cuarto y el tercer grados del Collège pertenecen al ciclo 4 de profundizaciones. La educación artística forma parte del marco del currículum nacional obligatorio $y$, para el caso de este nivel, incluye tres asignaturas: artes plásticas, educación musical e historia de las artes (Ministère de l'Education Nationale et de la Jeunesse, 2019). Asimismo, en los programas de todos los ciclos curriculares se destinan espacios para la educación artística, por una parte en las temáticas de las propias asignaturas del área de educación artística y cultural y, por otro, mediante la inclusión de elementos culturales y artísticos de manera transversal en el resto de las asignaturas (Tabla 4).

Además de las asignaturas obligatorias, durante este nivel los alumnos pueden participar en canto coral a fin de profundizar las competencias de la educación musical.

En cuanto a la carga horaria, la educación artística está prevista tanto en el horario escolar (una hora semanal) como en el extraescolar, a través de redes, proyectos, talleres, visitas a comunidades artísticas, entre otras actividades. Los objetivos de la educación artística y cultural en la educación obligatoria son tres: "permitir a todos los alumnos construir una cultura personal rica y coherente a lo largo de su trayecto escolar; desarrollar y reforzar su práctica artística; permitir el encuentro con artistas y las obras, la frecuentación de lugares culturales" (Ministère de l'Éducation Nationale et de la Jeunesse, 2018a:s/p).

De ahí que sean tres las dimensiones que se cultivan: las enseñanzas artísticas, los encuentros con artistas y obras, y las prácticas artísticas. Algunos de los contenidos de la formación artística y cultural son los siguientes. Para Artes Plásticas: la representación plástica y los dispositivos de presentación; las fabricaciones y la relación entre el objeto y el espacio. La materialidad de la producción plástica y la sensibilidad como constituyentes de la obra (Ministère de l'Éducation Nationale et de la Jeunesse, 2018b:s/p). En cuanto a las competencias que se deben alcanzarse, estas son: "Experimentar, producir, crear. Poner en marcha un proyecto artístico. Expresarse, analizar su práctica, y la de sus pares; establecer una relación con la de los artistas abrirse a la alteridad" (Ministère de l'Éducation Nationale et de la Jeunesse, 2018b:s/p). Para educación musical: desarrollo de los ámbitos de la producción y el de la percepción. Las competencias a desarrollar para el $6^{\circ}$ grado son: "Cantar e interpretar. Escuchar, comparar y comentar. Explorar, imaginar y crear. Intercambiar, compartir y argumentar" (Ministère de l'Éducation Nationale et de la Jeunesse, 2018b:s/p), en tanto que para los años restantes las competencias a desarrollar son: "Realizar proyectos musicales de interpretación o de creación. Escuchar, comparar, construir una 


\begin{tabular}{|c|c|c|c|c|}
\hline Asignado/Grado & $\begin{array}{c}\text { 6to } \\
\text { consolidación }\end{array}$ & $\begin{array}{l}\text { 5to } \\
\text { profundización }\end{array}$ & $\begin{array}{l}\text { 4to } \\
\text { profundización }\end{array}$ & $\begin{array}{l}\text { 3ro } \\
\text { profundización }\end{array}$ \\
\hline Francés & $4 h 30$ & $4 \mathrm{~h} 30$ & $4 \mathrm{~h} 30$ & $4 h$ \\
\hline Matemáticas & $4 \mathrm{~h} 30$ & $3 h 30$ & $3 \mathrm{~h} 30$ & $3 h 30$ \\
\hline $\begin{array}{l}\text { Historia-geografía } \\
\text { Enseñanza moral y } \\
\text { cívica }\end{array}$ & $3 \mathrm{~h}$ & $3 \mathrm{~h}$ & $3 \mathrm{~h}$ & $3 \mathrm{~h} 30$ \\
\hline Lengua viva 1 & $4 \mathrm{~h}$ & $3 h$ & $3 h$ & $3 h$ \\
\hline Lengua viva 2 & - & $2 \mathrm{~h} 30$ & $2 \mathrm{~h} 30$ & $2 \mathrm{~h} 30$ \\
\hline $\begin{array}{c}\text { Ciencias de la vida y } \\
\text { de la tierra }\end{array}$ & \multirow{3}{*}{$4 \mathrm{~h}$} & 1h 30 & 1h 30 & 1h 30 \\
\hline Ciencias físicas & & 1h 30 & 1h 30 & 1h 30 \\
\hline Tecnología & & 1h 30 & 1h 30 & 1h 30 \\
\hline $\begin{array}{c}\text { Educación física y } \\
\text { deportiva }\end{array}$ & $4 \mathrm{~h}$ & $3 \mathrm{~h}$ & $3 \mathrm{~h}$ & $3 \mathrm{~h}$ \\
\hline Artes plásticas & $1 \mathrm{~h}$ & $1 \mathrm{~h}$ & $1 \mathrm{~h}$ & $1 \mathrm{~h}$ \\
\hline Educación musical & $1 \mathrm{~h}$ & $1 \mathrm{~h}$ & $1 \mathrm{~h}$ & $1 \mathrm{~h}$ \\
\hline
\end{tabular}

Fuente: Ministère de l'Éducation Nationale et de la Jeunesse, 2016.

Tabla 4. Currículum del collège (educación secundaria obligatoria) en Francia.

Table 4. Curriculum of the collège (compulsory secondary education) in France.

cultura musical común. Explorar, imaginar, crear y producir. Intercambiar, compartir, argumentar y debatir" (Ministère de l'Éducation Nationale et de la Jeunesse, 2018b:s/p). Para el estudio de Historia de las artes, su enseñanza se vincula con el abordaje de los contenidos de todas las asignaturas. Entre los temas que se desarrollan se cuentan: Artes y sociedad en la época antigua y en la alta Edad Media. Formas y circulaciones artísticas (siglos IX-XV). Lo sagrado del artista (siglos XIV-XVII). El arte en el tiempo de las Luces y de las revoluciones (Ministère de l'Éducation Nationale et de la Jeunesse, 2018b:s/p). Las competencias a desarrollar son: "Opinar de manera argumentada sobre lo que representa o expresa una obra de arte. Analizar una obra de arte, por la observación o la escucha, sus principales características técnicas o formales. Situar: re- lacionar las características de una obra de arte a los usos, así como al contexto histórico y cultural de su creación. Orientarse en un museo, un lugar de arte, un sitio patrimonial" (Ministère de l'Éducation Nationale et de la Jeunesse, 2018b:s/p).

\subsection{Contenidos curriculares para la formación artística y cultural en México}

El currículum de la secundaria mexicana, puesto en marcha en 2018, está organizado a partir de tres componentes: aprendizajes clave, área de desarrollo personal y social, y ámbito de autonomía curricular. El primer elemento, aprendizajes clave, integra tres campos formativos: lenguaje y comunicación, pensamiento matemático, y exploración y comprensión del mundo natural y social. El segundo componente cu- 
rricular, desarrollo personal y social, incluye cuatro ámbitos de desarrollo: artístico y creatividad, corporal y salud, emocional y tutoría. El tercero, denominado autonomía curricular, alude al espacio que se ofrece a las escuelas para que, de acuerdo con sus necesidades, ocupen este para decidir qué se debe brindar a los alumnos, conforme a los cinco ámbitos establecidos: profundización en los aprendizajes clave, experiencias para el desarrollo personal y social, nuevos contenidos, contenidos regionales y local, proyectos de impacto social, Secretaría de Educación Pública (SEP, 2016) (Tabla 5).

El área de desarrollo artístico y creatividad forma parte del segundo componente curricular, y a semejanza de las áreas de desarrollo corporal y salud y desarrollo emocional, no está considerada como asignatura, puesto que con ella se trata de dar seguimiento al desarrollo integral de los alumnos y particularmente "al desarrollo de las capacidades de aprender a ser y aprender a convivir” (SEP, 2016, p.155).

Los objetivos planteados para esta área consisten en propiciar el desarrollo armónico de los alumnos, así como garantizar el derecho al acceso a la cultura y a la valoración del patrimonio artístico nacional e internacional. En el caso de desarrollo artístico y creatividad “...se incluye la experimentación, la exploración y el disfrute de diversas manifestaciones artísticas [...]. Sebusca propiciar espacios para que los alumnos desarrollen habilidades de expresión y apreciación artísticas, por lo que se pretende que los alumnos en el aula desplieguen procesos creativos a partir de retos, desafíos y estímulos que potencien la curiosidad, la iniciativa, la imaginación, la espontaneidad y la capacidad de disfrute" (SEP, 2016, p.157).

De los objetivos planteados para este referente en toda la educación básica se destacan los siguientes: identificar elementos y características de manifestaciones artísticas, explorar y experimentar con materiales de artes sonoras, plásticas, visuales, dancísticas y teatrales, manifestar una actitud creativa, resolver problemas en la creación de producciones artísticas, valorar la diversidad artística (SEP, 2016). Para el desarrollo de la educación artística y la creatividad se han definido los ejes de apreciación, expresión y contextualización, los cuales atraviesan toda la educación básica y se abordan en cuatro temáticas: artes plásticas y visuales, danza y expresión corporal, música y experimentación sonora, y, teatro y expresión corporal con voz. En el caso de la educación secundaria y con una carga horaria semanal de una hora, el desarrollo de estos tres ejes se ejemplifica con algunos de los objetivos prescritos en el currículum, como veremos a continuación.

1. Eje de apreciación.- a desarrollarse en las cuatro áreas con objetivos que van desde identificar elementos básicos de imágenes, hasta observar murales representativos para identificar sus características y temas. En danza y expresión corporal se propone desde experimentar secuencias de movimiento, hasta analizar técnicas de composición en la danza. En música y experimentación sonora, se pretende desde observar partituras y ejercitar la escritura musical, hasta identificar diferentes tipos de composición. En cuanto a teatro y expresión corporal con voz se parte de ubicar y dibujar áreas del escenario teatral para llegar a explorar técnicas usadas en la representación de emociones e ideas de una obra o expresión artística. 2. Eje de expresión.- de manera similar al primer eje, en este se ubican objetivos secuenciados. En el área de artes plásticas y visuales se parte de objetivos sencillos, tales como realizar un mural en el que se utilicen diferentes técnicas, hasta solicitar la elaboración grupal de una producción artística. En danza y expresión corporal, se propone desde elaborar una propuesta dancística, hasta la presentación de una puesta en escena en la que se especifiquen movimientos, objetos, niveles de participación, etc. Por lo que se refiere a música y experimentación sonora, se parte de lograr que el alumno interprete a través del canto diferentes piezas musicales y reconocer su intencionalidad, hasta la hechura de composiciones sencillas en un pentagrama, utilizando formas y texturas musicales. Final- 


\begin{tabular}{|c|c|c|c|c|c|}
\hline \multirow[t]{2}{*}{$\begin{array}{l}\text { COMPONENTE } \\
\text { CURRICULAR }\end{array}$} & \multicolumn{2}{|c|}{ ESTRUCTURA CURRICULAR } & \multicolumn{3}{|c|}{ GRADO ESCOLAR } \\
\hline & & & $1^{\mathrm{o}}$ & $2^{\circ}$ & $3^{\circ}$ \\
\hline \multirow[t]{8}{*}{ Aprendizajes clave } & $\begin{array}{l}\text { Campos } \\
\text { formativos y } \\
\text { asignaturas }\end{array}$ & $\begin{array}{l}\text { Lenguaje y comu- } \\
\text { nicación }\end{array}$ & \multicolumn{3}{|c|}{ Lengua materna y literatura } \\
\hline & & & \multicolumn{3}{|c|}{ Lengua extranjera (inglés) } \\
\hline & & & $\begin{array}{l}\text { Pensamientos } \\
\text { matemáticos }\end{array}$ & \multicolumn{2}{|c|}{ Matemáticas } \\
\hline & & & $\begin{array}{l}\text { Exploración del } \\
\text { mundo natural y } \\
\text { social }\end{array}$ & \multicolumn{2}{|c|}{ Ciencias y tecnología } \\
\hline & & & Biología & Física & Química \\
\hline & & & \multicolumn{3}{|c|}{ Historia } \\
\hline & & & Geografía & \multicolumn{2}{|c|}{ 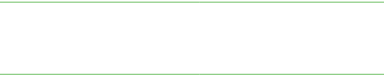 } \\
\hline & & & \multicolumn{3}{|c|}{ Formación cívica y ética } \\
\hline \multirow[t]{3}{*}{$\begin{array}{l}\text { Desarrollo personal y } \\
\text { social }\end{array}$} & Áreas & $\begin{array}{l}\text { Desarrollo corpo- } \\
\text { ral y salud }\end{array}$ & \multicolumn{3}{|c|}{ Desarrollo corporal y salud } \\
\hline & & $\begin{array}{l}\text { Desarrollo artísti- } \\
\text { co y creatividad }\end{array}$ & \multicolumn{3}{|c|}{ Desarrollo artístico y creatividad } \\
\hline & & $\begin{array}{c}\text { Desarrollo emo- } \\
\text { cional }\end{array}$ & \multicolumn{3}{|c|}{ Orientación y tutoría } \\
\hline \multirow[t]{5}{*}{ Autonomía curricular } & Ámbitos & $\begin{array}{l}\text { Profundización de } \\
\text { aprendizajes clave }\end{array}$ & \multicolumn{3}{|c|}{$\begin{array}{l}\text { Definición a cargo de la escuela con base } \\
\text { en lineamientos específicos por la Secre- } \\
\text { taría de Educación Pública }\end{array}$} \\
\hline & & $\begin{array}{l}\text { Ampliación de las } \\
\text { oportunidades } \\
\text { para el desarrollo } \\
\text { personal y social }\end{array}$ & & & \\
\hline & & $\begin{array}{l}\text { Nuevos contenidos } \\
\text { relevantes }\end{array}$ & & & \\
\hline & & $\begin{array}{l}\text { Conocimiento de } \\
\text { contenidos regio- } \\
\text { nales y locales }\end{array}$ & & & \\
\hline & & $\begin{array}{l}\text { Impulso a pro- } \\
\text { yectos de impacto } \\
\text { social }\end{array}$ & & & \\
\hline
\end{tabular}

Fuente: Ministère de l'Éducation Nationale et de la Jeunesse, 2016.

Tabla 5. Currículum de la educación secundaria obligatoria en México.

Table 5. Curriculum of compulsory secundary education in Mexico. 
mente, en teatro y expresión corporal con voz se parte desde representar objetos, animales y personajes, hasta la ejecución de una improvisación. 3. Eje de contextualización.- en relación con artes plásticas, los objetivos van desde ser capaz de interpretar los componentes de diversas imágenes para interpretar sus significados hasta lograr el registro del proceso creativo a través de fotografías o dibujos. En el área de danza y expresión corporal se parte de reconocer la función social de la danza, hasta el reconocimiento de las diferentes manifestaciones artísticas colectivas de México. En cuanto a la música y la experimentación sonora, se parte de tener capacidad para inferir la intención de sonidos e instrumentos musicales de una pieza musical, hasta de representar una pieza musical propia a partir de una temática social. En cuanto a teatro y expresión corporal con voz, se inicia reconociendo la intención de una obra teatral, hasta proponer el análisis de diversas obras teatrales.

\subsection{Formación de docentes para la educación artística y la cultura en Francia}

La formación profesional y la formación para la enseñanza están a cargo de las Escuelas Superiores del Profesorado y de la Educación (ESPE), pertenecientes a diversas universidades francesas distribuidas en todas las academias del territorio francés. La oferta académica de las ESPE incluye opciones de formación en el campo de la educación artística y cultural; para el ingreso a estas instituciones se requiere de inicio contar con una licenciatura de cualquier rama de las humanidades y las artes, además de presentar un concurso de oposición que permita el acceso al nivel de maestría o Máster. Cabe señalar que cada universidad ofrece diversas opciones de formación artística a nivel de licenciatura, así como de ingreso al Máster para la formación docente. De entre las diferentes opciones para la licenciatura en artes y cultura se mencionan las de Artes Plásticas Historia del Arte, Musicología, Artes del Espectáculo, Artes y Cultura, Cine, Moda y altas tecnologías, Animación y valoración del Patrimonio, Acciones culturales y profesiones de la Arqueología, Téc- nicas del Sonido y de la Imagen, profesiones de la Exposición y Tecnologías de la Información, las cuales se imparten en más de veinte universidades. Así por ejemplo, en la Universidad de Picardie Jules Verne, se ofrecen las licenciaturas en Mención Artes del espectáculo: Estudios Cinematográficos y Estudios Teatrales; Mención Artes Plásticas: Artes Plásticas y Comics; Técnicas del Sonido y de la Imagen (Université de Picardie Jules Verne, s/f). En la Universidad Panthéon Sorbonne, Paris I, se ofrecen las siguientes licenciaturas con dos menciones: a) Mención Artes Plásticas: Artes Plásticas, Diseño, Artes y Medios de Comunicación, Estética y Ciencias del Arte, Profesiones de las Artes y de la Cultura, y b) Mención Cine: Cine: práctica y estética, y Cine-Gestión (Université Paris I, Panthéon Sorbonne, s/f).

Para formarse como profesor de educación artística para el Collège, una vez concluida la licenciatura se requiere optar por la Mención de segundo grado, la cual posibilita el ingreso a la docencia tanto en el Collège como en el Liceo. En cuanto a los estudios del Máster, una parte del currículum es común y requiere ser cursado por todos los estudiantes de las ESPE. Asimismo, desde el primer año de esta especialidad los alumnos realizan prácticas de observación y docencia en las escuelas; y al finalizar este primer período están obligados a presentar el concurso de oposición para ingresar al segundo año, a partir del cual los estudiantes se convierten en funcionarios practicantes y obtienen una remuneración equivalente a la de un profesor de tiempo completo. En el caso de la opción del Máster en artes, a partir del segundo año las ESPE ofrecen opciones de acceso a diversas universidades, particularmente para las asignaturas de Artes Plásticas, Música e Historia de las Artes.

\subsection{Formación de docentes para la educación artística y la cultura en México}

Las condiciones para la formación de docentes en educación artística en México revelan una situación de dispersión y debilidad en los pro- 
cesos de formalización, toda vez que la preparación en cualquiera de las artes se realiza en diferentes instituciones, públicas y privadas y con diferentes niveles de reconocimiento oficial, lo mismo para formar músicos, danzantes, actores, cineastas, creadores en artes plásticas y visuales, o bien, para la formación de docentes en esta área. Así por ejemplo, al igual que en varias universidades públicas del país, en el caso de la Universidad Nacional Autónoma de México (UNAM) se cuenta con diversas dependencias para la formación en artes, como son: la Escuela Nacional de Artes Cinematográficas que forma cineastas en distintas ramas de la expresión audiovisual; el Centro Universitario de Teatro que forma artistas de la escena; la Escuela Nacional de Danza Clásica y Contemporánea que forma bailarines profesionales altamente calificados; la Facultad de Artes y Diseño que forma técnicos profesionales en producción de imagen digital y licenciados en arte y diseño, capaces de plantear soluciones a problemas de comunicación visual; la Facultad de Filosofía y Letras que ofrece una formación teatral como fenómeno artístico (Universidad Nacional Autónoma de México, 2018).

Por otra parte, se cuenta con la preparación brindada por el Instituto Nacional de Bellas Artes y Literatura, dependiente de la Secretaría de Cultura y que, en vinculación con el Centro Nacional de las Artes, cuenta con una oferta institucional diversificada con opciones formativas en artes. Así por ejemplo, la Escuela Nacional de Danza Clásica y Contemporánea ofrece licenciaturas diversas; las Escuelas de Artes Plásticas y Visuales: Artesanías, Diseño y La Esmeralda, ofrecen formación en las áreas de Pintura, Escultura y Grabados; la Escuela Nacional de Arte Teatral que forma profesionales del teatro; la Escuela Superior de Música y el Conservatorio Nacional de Música que ofrecen formaciones diversas en el nivel medio superior y superior; y el Centro de Capacitación Cinematográfica, para la preparación de los cineastas (Universidades de México, 2019).

En este contexto observamos que en México hay una amplia variedad de instituciones públicas y privadas que forman artistas en diferentes disciplinas artísticas. Por ejemplo, en el caso de la formación de actores las opciones tanto de instituciones públicas como privadas en la Ciudad de México son variadas, ubicando así: la UNAM con la licenciatura en Actuación en el Centro Universitario de Teatro, y en la Facultad de Filosofía y Letras con la licenciatura en Literatura Dramática y Teatro; la Escuela Nacional de Arte Teatral, con la licenciatura en Actuación y en Escenografía; la Casa del Teatro con la licenciatura en Actuación; la Universidad Autónoma del Estado de México, con la licenciatura en Artes Teatrales; la Universidad Autónoma del Estado de Morelos, con la licenciatura en Teatro; la Benemérita Universidad Autónoma de Puebla, con la licenciatura en Arte Dramático; la Universidad Veracruzana, con la licenciatura en Teatro; la Universidad Autónoma de Nuevo León, con la licenciatura en Arte Teatral; la Escuela Superior de Artes de Yucatán, con la licenciatura en Teatro (Universidades de México, 2019).

Por lo que se refiere a la formación en música y de manera similar a lo que sucede con la de arte, la oferta académica incluye diversas instituciones públicas y privadas, con un claro predominio de las instituciones públicas universitarias, tanto en la Ciudad de México como en los diferentes estados de la república mexicana. Así por ejemplo, la Universidad Anáhuac ofrece la licenciatura en Música Contemporánea; la UNAM ofrece múltiples opciones como son las licenciaturas en Etnomusicología, Canto, en Composición, en Educación Musical, en Música Instrumentista, en Piano; la Universidad de Guadalajara con la licenciatura en Música; la Universidad Autónoma de Nuevo León también cuenta con varias ofertas de licenciaturas en música: Música e Instrumentista, Música y Director de Coros, Música y Cantante, Música y Educación Musical, Música y Composición; la Universidad Autónoma de Baja California, la Universidad Autónoma de Ciudad Juárez, la Universidad de Sonora y la Universidad Autónoma de Chihuahua ofrecen una Licenciatura en Música, pero también las universidades autónomas de Nayarit, Colima, 
Sinaloa, Guanajuato, Estado de Hidalgo, Estado de México, Tamaulipas, Michoacana de San Nicolás de Hidalgo, Aguascalientes; la Universidad Autónoma de Coahuila cuenta con la oferta de varias licenciaturas: en Música con Acentuación en Canto, en Composición, en Cuerdas, y en Piano; el Conservatorio de Las Rosas, en Michoacán, ofrece una licenciatura en Música con varias opciones: Área de Canto, Área de Clarinete, Área de Composición, Área de Contrabajo, Área de Corno Francés. Igualmente cuenta con programas de licenciatura en Percusiones, en Piano, en Saxofón, en Trombón, en Trompeta, en Tuba, en Viola, en Fagot, en Violoncello, en Violín, en Musicología, en Guitarra, en Flauta, y en Oboe; la Escuela Superior de Artes de Yucatán ofrece la licenciatura en Artes Musicales; la Universidad Autónoma de Zacatecas cuenta con la licenciatura en Música con Énfasis en Instrumento y la licenciatura en Canto; la Universidad de Ciencias y Artes de Chiapas tiene programas de licenciatura en Música, en Jazz y Música Popular; la Universidad de Las Américas de Puebla ofrece la licenciatura en Música; por último, la Universidad Autónoma de Querétaro ofrece la licenciatura en Música Popular Contemporánea.

No obstante esta multivariada oferta de carreras en la disciplina de la música, el número de instituciones con opciones de formación docente en educación musical es muy reducida. Así por ejemplo, este tipo de preparación solo se ofrece en la Facultad de Música de la UNAM, en la Universidad Juárez del Estado de Durango, en la Universidad Veracruzana y en la Universidad Autónoma de Coahuila (con la Licenciatura en Educación Musical). De hecho, observamos que en el panorama nacional son muy escasas las instituciones que cuentan con programas específicos para la formación docente. El INBAL tiene en danza dos licenciaturas de Danza Clásica, una orientada hacia la docencia y la otra con un plan especial para hombres (Gobierno de México, Secretaría de Cultura, s/f-a); el Centro Nacional de las Artes ha creado recientemente dos diplomados: 1) el Diplomado Interdisciplinario para la Enseñanza de las Artes en Educación Básica diseñado para que “...los profesores adquieran herramientas de indagación, reflexión e integración que los aproxime a los procesos artísticos y su vinculación con las diferentes áreas del conocimiento mediante tres ejes de experimentación y reflexión: cuerpo, espacio y tiempo" (Gobierno de México, Secretaría de Cultura, s/fb:s/p); y, 2) el Diplomado semipresencial para el apoyo a la Enseñanza de las Artes en la Educación Básica, el cual está destinado a los profesionales del arte y de la educación a fin de coadyuvar en la formación de docentes en las artes, haciendo uso de las tecnologías como medios de aprendizaje.

\section{CONCLUSIONES}

A manera de cierre para el presente artículo incluimos una revisión sintética de la aproximación comparativa realizada, mediante la cual nos interesa destacar los siguientes hallazgos agrupados en cinco rubros (Tabla 6). Un primer rubro tiene que ver con la ponderación atribuida a la formación académica en educación artística y cultura en el nivel de educación secundaria obligatoria, y de acuerdo con la perspectiva internacional puntualizada en documentos y textos internacionales (Jiménez, 2006; UNESCO, 2006; EACEA P9/Eurydice, 2009; Ferro et al, 2019), observamos que en el discurso oficial de ambos países hay congruencia con los planteamientos universalizantes sobre la importancia de la sensibilización a las artes y la cultura durante la etapa de la educación obligatoria, valorando necesariamente la relevancia de la dimensión pedagógica de las artes y la cultura para la educación formal y no formal de niños y jóvenes. En este sentido, ambos casos nacionales son representativos de la universalización de la educación secundaria obligatoria en el entramado de complejas tendencias mundiales de internacionalización, obligatoriedad y estandarización de la oferta educativa. Como segundo rubro ubicamos lo concerniente a las políticas públicas implantadas por cada país, encontrando diferencias importantes. En el caso de Francia, mediante la política educativa se ha posicionado la educación artística y cul- 


\begin{tabular}{|c|c|c|}
\hline Rubros/País & Francia & México \\
\hline $\begin{array}{l}\text { VINCULACIÓN CON } \\
\text { EL PANORAMA } \\
\text { INTERNACIONAL }\end{array}$ & \multicolumn{2}{|c|}{$\begin{array}{l}\text { Congruencia del discurso oficial con planteamientos universalizantes sobre la } \\
\text { importancia de la sensibilización de niños y jóvenes para las artes y la cultura } \\
\text { mediante de la educación obligatoria, valorando la relevancia de la dimensión } \\
\text { pedagógica. }\end{array}$} \\
\hline $\begin{array}{l}\text { POLÍTICAS } \\
\text { PÚBLICAS }\end{array}$ & $\begin{array}{l}\text { Posicionamiento delaeducaciónartística } \\
\text { y cultural como una prioridad vinculada } \\
\text { con procesos de democratización, } \\
\text { garantizando igualdad de acceso a } \\
\text { las prácticas artísticas y culturales } \\
\text { dentro y fuera del ámbito escolar. }\end{array}$ & $\begin{array}{l}\text { Persistencia de limitantes de ín- } \\
\text { dole diversa para el acceso al arte } \\
\text { ylacultura, en específico dela po- } \\
\text { blación socialmente marginada. }\end{array}$ \\
\hline $\begin{array}{l}\text { ORIENTACIÓN } \\
\text { FORMATIVA DEL } \\
\text { CURRÍCULUM }\end{array}$ & $\begin{array}{l}\text { planteada como un eje de formación } \\
\text { académica queatraviesadesdela escuela } \\
\text { maternal hasta el liceo, buscando } \\
\text { el desarrollo de la sensibilidad, del } \\
\text { pensamiento crítico y de la creatividad. }\end{array}$ & $\begin{array}{l}\text { La educación artística y cultu- } \\
\text { ral está orientada al logro de un } \\
\text { desarrollo armónico, vinculado } \\
\text { con el principio de respaldar el } \\
\text { derecho al acceso a la cultura y a } \\
\text { la valoración del patrimonio ar- } \\
\text { tístico nacional e internacional. }\end{array}$ \\
\hline $\begin{array}{l}\text { ESTRUCTURA } \\
\text { CURRICULARY } \\
\text { PONDERACIÓN } \\
\text { ACADÉMICA }\end{array}$ & $\begin{array}{l}\text { Organización curricular a partir de } \\
\text { un zoclo común de conocimientos, } \\
\text { competencias y cultura, en la cual la } \\
\text { educación artística se vincula con los } \\
\text { ámbitos de lenguajes, sistemas naturales } \\
\text { ytécnicos, yrepresentaciones del mundo } \\
\text { y de la actividad humana. Se pondera } \\
\text { una formación académica basada } \\
\text { en una perspectiva interdisciplinar. }\end{array}$ & $\begin{array}{l}\text { Marco curricular diseñado a } \\
\text { partir de los ejes de aprecia- } \\
\text { ción, expresión y contextua- } \\
\text { lización. El abordaje de los } \\
\text { contenidos de la educación } \\
\text { artística se ha distribuido en } \\
\text { temáticas básicas sobre las ar- } \\
\text { tes plásticas, música y teatro. }\end{array}$ \\
\hline $\begin{array}{l}\text { OFERTA } \\
\text { ACADÉMICA PARA } \\
\text { LA FORMACIÓN } \\
\text { DOCENTE }\end{array}$ & $\begin{array}{l}\text { Oferta formativa de licenciaturas y } \\
\text { maestrías de alto nivel, distribuida en } \\
\text { todo el territorio nacional. Esfuerzo } \\
\text { gubernamental decidido para garanti- } \\
\text { zar la calidad de la preparación de } \\
\text { los formadores. }\end{array}$ & $\begin{array}{l}\text { Multivariedad de la oferta for- } \\
\text { mativa en instituciones públi- } \\
\text { cas y privadas distribuida en } \\
\text { todo el país. Pendiente de lo- } \\
\text { grar una infraestructura aca- } \\
\text { démico-administrativa ho- } \\
\text { mogénea para la formación de } \\
\text { plantas docentes acordes con el } \\
\text { nivel de educación secundaria. }\end{array}$ \\
\hline
\end{tabular}

Fuente: Elaboración de las autoras.

Tabla 6. Comparativo de formación artística en educación secundaria obligatoria.

Table 6. Comparison of artistic training in compulsory secondary education. 
tural como una prioridad aparejada a procesos de democratización que garanticen igualdad de acceso a las prácticas artísticas y culturales para todos en el ámbito escolar y fuera de él. En contraste, en el caso de México aún prevalecen múltiples obstáculos en diferentes ámbitos para que la población en su totalidad tenga acceso al arte y a la cultura, principalmente aquellos grupos sociales marginados, situación que evidencia la desigualdad prevaleciente en nuestra sociedad.

Un tercer rubro es el concerniente a la orientación formativa estipulada en el currículum obligatorio, en concreto en la educación artística y cultural. En el caso francés esta formación atraviesa desde la escuela maternal hasta el liceo y se centra en la enseñanza del arte y la cultura con el complemento disciplinario del teatro, la música y la danza, buscando el desarrollo de la sensibilidad, del pensamiento crítico y de la creatividad. En el caso mexicano la educación artística y cultural se orienta a lograr un desarrollo armónico, vinculado con el principio de respaldar el derecho al acceso a la cultura y a la valoración del patrimonio artístico nacional e internacional. Ligado al anterior, el cuarto rubro incluye dos aspectos también importantes como son la estructura curricular y con ella la ponderación académica del área respectiva. En Francia el currículum de la educación obligatoria está organizado a partir de un zoclo común de conocimientos, competencias y cultura, y en dicha estructura la educación artística se vincula con tres de los ámbitos del zoclo común: lenguajes, sistemas naturales y técnicos, y representaciones del mundo y de la actividad humana, por lo que la formación propuesta se ha ponderado con base en una perspectiva interdisciplinar. En México el marco curricular de toda la educación cuenta con los ejes de apreciación, expresión y contextualización, a partir de los cuales se abordan los contenidos de la educación artística distribuidos en temáticas básicas sobre las artes plásticas, música y teatro.

El quinto y último rubro retoma lo concerniente a la oferta académica para la formación de docentes y el estrecho vínculo con la política educativa de cada país, dado que dicha formación alude directamente al papel del Estado como responsable de la educación oficial. En el caso de Francia la oferta de formación docente está distribuida en todo el territorio nacional, con universidades e instituciones de educación superior (ESPE) que ofertan licenciaturas y maestrías con alto nivel de rigurosidad tanto para el ingreso como para la certificación, demostrando el esfuerzo decidido de los gobiernos franceses de las últimas décadas por asegurar lo mismo la calidad de la preparación de los formadores como la de los estudiantes, a partir de una visión de proyectos creativos y de reconocimiento e integración con otras perspectivas culturales. En el caso de México y a pesar de la multivariedad de la oferta formativa en instituciones públicas y privadas asentadas a lo largo y ancho de país, las políticas gubernamentales sobre la preparación de docentes de las últimas décadas aún no han logrado consolidar una infraestructura académico-administrativa homogénea para la formalización de este proceso que garantice contar con plantas docentes consolidadas acordes con la calidad requerida para el nivel de educación secundaria.

En todo caso, el precedente señalado al inicio de este documento sobre la vinculación del panorama educativo de cada país con el respetivo contexto sociodemográfico nos ayuda a comprender las condiciones de avance o estancamiento prevalecientes en los casos analizados, además de ubicar la importancia de la aproximación comparativa para situar en perspectiva las semejanzas y las diferencias entre Francia y México, por cuanto a la contribución formativa de la educación artística y cultural en la niñez y la juventud del siglo XXI.

La elaboración de este artículo se concretó en el marco del Proyecto de Investigación Institucional UNAM-DGAPA- PAPIIT IN401218 "La educación secundaria europea y latinoamericana. Un estudio comparado”. En este sentido expresamos nuestro agradecimiento por el wapoyo institucional recibido. 


\section{REFERENCIAS}

Adamson, B., y Morris, P. (2010). "La comparación de currículos”. En M. Bray, B. Adamson, y M. Mason, Educación comparada. Enfoques y métodos (pp. 321342). Buenos Aires: Granica.

Aguirre, I. (2008). Las artes en la trama de la cultura. Fundamentos para renovar la educación artística, en Revista Digital do LAV, 1(1) Recuperado de http:// www.redalyc.org/articulo.oa?id=337027033002. Fecha de consulta: 10 de octubre de 2019.

Aguirre, I., y Giráldez, L. (2009). "Fundamentos curriculares de la educación artística”. En L. Jiménez, I. Aguirre, \& L. Pimentel, Educación artística, cultura y ciudadanía (pp. 75-87). Madrid: OEI. Recuperado de https://www.oei.es. Fecha de consulta: 10 de octubre de 2019.

Banco Mundial (2019). Datos estadísticos: Francia y México. Estados Unidos de Norteamérica: Banco Mundial. Recuperado de http://datos.bancomundial. org/. Fecha de consulta: 19 de octubre de 2019.

Casimiro, A. (2015). "¿Todavía es posible hablar de un currículum politico?”. En A. Alba, y A. Casimiro, Diálogos curriculares entre México y Brasil (pp. 4361). México: UNAM/IISUE.

Central Intelligence Agency (2019). The world factbook. Francia y México. Estados Unidos de Norteamérica: CIA. Recuperado de https://www.cia.gov/ library/publications/the-world-factbook/. Fecha de consulta: 10 de octubre de 2019.

EACEA P9/Eurydice (2009). Arts and Cultural Education at School in Europe. Bruselas: EACEA. Recuperado de http://publications.europa.eu/resource/cellar/4a035c44-0231-4329-9150c1bf07132756.0001.01/DOC_1. Fecha de consulta: 15 de mayo de 2019.

Enel, F. (2011). Politiques d'éducation artistique et culturelle: rôle et action des collectivités locales. In Culture, Études. Politiques publiques et régulation, République Française: Secrétariat Général, Paris. Recuperado de https://www.cairn.info/revue-culture-etudes-2011-2.htm. Fecha de consulta: 20 de junio de 2019.

Ferro, L., Wagner, E., Veloso, L., IJdens, T., \& Teixeira Lopes, J. (2019), Arts and Cultural Education in a World of Diversity ENO Yearbook 1, Suiza: Springer (eBook). Recuperado de https://doi.org/10.1007/9783-030-06007-7. Fecha de consulta: 15 de octubre de 2019.

Gobierno de México, Secretaría de Cultura (s/f-a). Escuela Nacional de Danza Clásica y Contemporánea. Recuperado de https://endcc.inba.gob.mx/. Fecha de consulta: 23 de junio de 2019.

Gobierno de México, Secretaría de Cultura (s/f-b). Centro Nacional de las Artes. Recuperado de https:// www.cenart.gob.mx/vida-academica/programas-permanentes/programa-de-formacion-docente-en-elcampo-de-las-artes/. Fecha de consulta: 23 de junio de 2019.

Gobierno de la República, Secretaría de Educación Pública, Secretaría de Cultura (2017). Modelo Educativo. Cultura en tu escuela. Recuperado de https://www. gob.mx/cms/uploads/attachment/file/283756/CULTURA_DIGITAL.pdf. Fecha de consulta: 23 de junio de 2019.

Jiménez, L., Aguirre, I., y Pimentel, L. (2009). "Introducción”. En L. Jiménez, I. Aguirre, \& L. Pimentel, Educación artística, cultura y ciudadanía (pp. 11-14). Madrid: OEI. Recuperado de https://www.oei.es. Fecha de consulta: 10 de octubre de 2019.

Ministère de l'Éducation Nationale et de la Jeunesse (2018a). L'éducation artistique. De la maternelle au baccalauréat. Recuperado de https://www.education. gouv.fr/cid20725/l-education-artistique-et-culturelle.html\#Objectifs_de l'éducation artistique et culturelle

Ministère de l'Éducation Nationale et de la Jeunesse (2018b). Les programmes du collège. Recuperado de ttps://www.education.gouv.fr/cid81/les-programmes-du-college.html. Fecha de consulta: 20 de junio de 2019.

Ministère de l'Education Nationale et de la Jeunesse (2019). Le socle commun de connaissances, de compétences et de culture. Recuperado de https://eduscol. education.fr/cid86943/le-socle-commun.html. Fecha de consulta: 20 de junio de 2019.

OECD, PISA, Organización para la Cooperación y el Desarrollo Económicos, PISA (2018). Database. Recuperado de http://www.oecd.org/pisa/data/2018database/. Fecha de consulta: 24 de mayo de 2019.

Presidencia de la República (2019). Plan Nacional de Desarrollo 2019-2024. Recuperado de https://lopezobrador.org.mx/wp-content/uploads/2019/05/ PLAN-NACIONAL-DE-DESARROLLO-2019-2024. pdf. Fecha de consulta: 25 de junio de 2019.

République Française. Ministère de l'Éducation Nationale, Ministère de la Culture et de la Communication et autres (2016). Charte pour l'Éducation Artistique et culturelle. À l'Initiative du Haut Conseil de l'Éducation Artistique et Culturelle. Recuperado de https://www.education.gouv.fr/l-education-artistique-et-culturelle-7496. Fecha de consulta: 20 de junio de 2019. 
République Française. Ministère de la Culture (s/f). Éducation artistique et culturelle. Recuperado de http://www.culture.gouv.fr/Thematiques/Education-artistique-et-culturelle.

Ruiz, C., y Santos, M. (2009). "La educación artística: itinerario de la agenda internacional”. In L. Jiménez, I. Aguirre, \& L. Pimentel, Educación artística, cultura y ciudadanía (pp. 155-192). Madrid: OEI. Recuperado de https://www.oei.es. Fecha de consulta: 23 de junio de 2019.

Schriewer, J. (1993). "El método comparativo y la necesidad de externalización: criterios metodológicos y conceptos sociológicos”. En J. Schriewer, y F. Pedró, Manual de educación comparada. Teorias, investigaciones, perspectivas pp. 189-251). Barcelona: Pomares-Corredor.

Schriewer, J. (2011). "Sistema mundial y redes de interrelación: la internacionalización de la educación y el papel de la investigación comparada”. En M. Caruso, y H-E. Tenorth, Internacionalización. Politicas educativas y reflexión pedagógica en un medio global (pp. 41-105). Buenos Aires: Granica.

Secretaría de Cultura (2015). Ejes de la política cultural. Recuperado de https://www.cultura.gob.mx/ ejes/. Fecha de consulta: 23 de junio de 2019.

SEP, Secretaría de Educación Pública (2016). Propuesta curricular para la educación obligatoria 2016. Recuperado de https:/www.gob.mx/cms/uploads/ docs/Propuesta-Curricular-baja.pdf. Fecha de consulta: 23 de junio de 2019.

Sistema de Información de Tendencias Educativas en América Latina (Siteal) (2019). México, Educación. Documento de país. Chile: UNESCO, IIPE. Recuperado de http://www.siteal.iipe.unesco.org/sites/default/ assets/pdf/educacion/siteal_ed_mexico_20180802. pdf. Fecha de consulta: 24 de mayo de 2019.
UNESCO, Organización de las Naciones Unidas para la Educación, la Ciencias y la Cultura (2006). Hoja de Ruta para la Educación Artística. Recuperado de http://www.unesco.org/new/fileadmin/MULTIMEDIA/HQ/CLT/CLT/pdf/Arts_Edu_RoadMap_ es.pdf. Fecha de consulta: 24 de mayo de 2019.

UNESCO/Cine, Organización de las Naciones Unidas para la Educación, la Ciencias y la Cultura (2011). Clasificación Internacional Normalizada de la Educación (CINE). Recuperado de https://unesdoc.unesco. org/ark:/48223/pf0000220782. Fecha de consulta: 24 de mayo de 2019.

Université de Picardie Jules Verne (s/f.). Catalogue de formation. Recuperado de https:// www.u-picardie.fr/catalogue-formations/FI/co/ catalogueUPJV_FI.html?query $=\&$ filters $=\% 5 \mathrm{~B} \%$ 5 D\&order $=$ titleRaw\&orderType $=$ asc $\&$ page $=0 \&$ ho mecodes=composantes\%20typeDiplome\%20mentions\%20titleRaw. Fecha de consulta: 17 de julio de 2019.

Université Paris I, Panthéon Sorbonne (s/f). Licences. Recuperado de https://www.pantheonsorbonne. fr/ufr/ufr04/loffre-de-formation/licences/. Fecha de consulta: 17 de julio de 2019.

Universidad Nacional Autónoma de México (2018). Oferta educativa nivel Licenciatura, México, Dirección General de Administración Escolar. Recuperado de https://www.dgae.unam.mx/planes/licenciatura.html. Fecha de consulta: 17 de julio de 2019.

Universidades de México (2019). Carreras y Licenciaturas en Música. Recuperado de https://universidadesdemexico.mx/carreras/licenciatura-en-musica. Fecha de consulta: 17 de julio de 2019. 